\title{
A Survey on Novel Estimation Approach of Motion Controllers for Self-Driving Cars
}

\author{
Mr. R. Vinothkanna \\ Department of ECE, \\ Vivekanandha College of Technology for Women, \\ Namakal, India. \\ rvinothkannaphd@gmail.com
}

\begin{abstract}
The motion planning framework is one of the challenging tasks in autonomous driving cars. During motion planning, predicting of trajectory is computed by Gaussian propagation. Recently, the localization uncertainty control will be estimating by Gaussian framework. This estimation suffers from real time constraint distribution for (Global Positioning System) GPS error. In this research article compared novel motion planning methods and concluding the suitable estimating algorithm depends on the two different real time traffic conditions. One is the realistic unusual traffic and complex target is another one. The real time platform is used to measure the several estimation methods for motion planning. Our research article is that comparing novel estimation methods in two different real time environments and an identifying better estimation method for that. Our suggesting idea is that the autonomous vehicle uncertainty control is estimating by modified version of action based coarse trajectory planning. Our suggesting framework permits the planner to avoid complex and unusual traffic (uncertainty condition) efficiently. Our proposed case studies offer to choose effectiveness framework for complex mode of surrounding environment.
\end{abstract}

Keywords: Estimation methods, Motion controllers, Artificial Intelligence

\section{Introduction}

Deep learning and Artificial Intelligent is an emerging trend for self-driving cars. Many developed countries having advancement in car manufacturing with learning control algorithm. Self-driving car springs from traffic congestion problem [1]. Now a day, self-driving cars start to migrate from research testing level to driving on public roads in many countries. Logically, it will decrease road accidents. Mainly the autonomous driving focusing the problem solving area classical perception, path planning and motion control methods which are shown in the figure 1. There are two type of path planning named low level and high level. The figure 2 shows the high level path planning by driving car. This path planning comes under motion controller procedure. The longitudinal and lateral steering commands of vehicle will be computed by motion controller. With some fixed parameters, the priori model had designed in earlier [2].

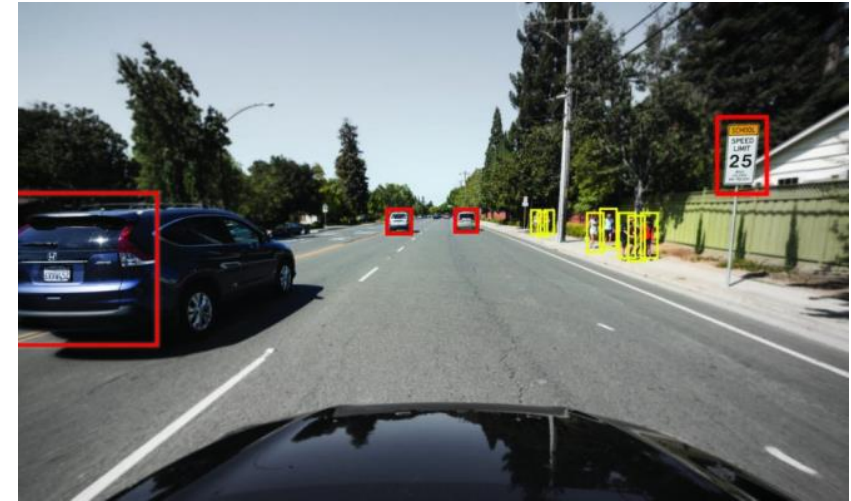

Figure 1 Perception control Unit of Self driving car 
Journal of Electronics and Informatics (2020)

Vol.02/ No.04

Pages: 211-219

https://www.irojournals.com/iroei/

DOI: https://doi.org/10.36548/jei.2020.4.003

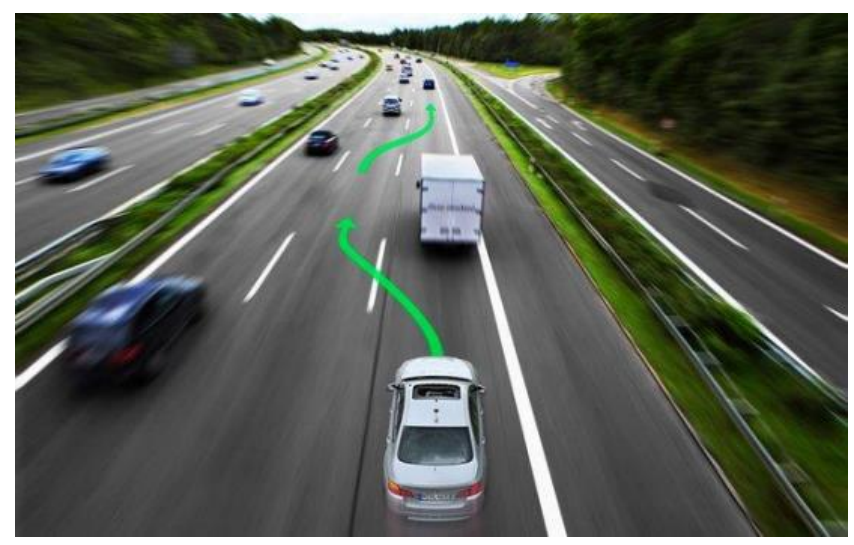

Figure 2 Path planning by Self driving car

So the autonomous system fails in many complex environments especially with motion controller architecture. Recently, the gathering data is well training with deep learning and approximation of the true system model will be good efficiency. The traffic problem and death rate by road accident by cars increases day by day in several countries [3]. Due to human mistakes and diversion, the traffic accidents are happening. So the main aim is to improve the safety during vehicle travel.

\section{Organization of the Research}

This research article covers introduction, literature survey, with problem statement and proposing solution, theoretical approach and discussion for estimating the motion controllers for autonomous vehicle. Finally, conclusion with future improvement discussed.

\section{Related Works}

The motion planning algorithms have used widely for autonomous vehicle. For the autonomous vehicle, the lattice planner technique is used for low traffic conditions [4]. McNaughton et al [5] introduces spatio temporal lattice trajectory planning for high way driving vehicle. They limited in their algorithm with priori planning methods. So Xu et al. [6] and Gu et al.[7] investigate by improved version of spatio-temporal lattice for posterior optimization and extra feature search respectively. Moreover, these planning methods are sensing prediction of obstacles and deterministic motion in real time environment. The author [8] introduces a Partially Observable Markov Decision Process (POMDP) is used to find the probability distribution (PD) in all the possible states. When the problem dimension increases this algorithm leads control unit in to hard up. With the help of Kalman filter, there is an estimation technique to discover the vehicles state and uncertainty. This motion planning algorithm is termed Linear Quadratic Regulator (LQR) which will find the optimal trajectory $[9,10]$. Based on the reachable set, the autonomous vehicle will get motion planning accurate with verification techniques. Here the planned trajectory is drawn as safe when no overlap between reachable set of obstacles and Autonomous Vehicle (AV) [11]. Based on the Markov chain, the estimation of the prediction for possible behaviors of traffic is computed successfully. But this method fails in the unsafe condition of autonomous vehicle due to no prior knowledge of trajectory is unsafe and priori feedback gave wrong command to the planner [12]. Here safe trajectory is not possible during unsafe condition of AV. The author considered Point based Markov Decision Process (QMDP) [13] above mentioned uncertainty problems of AV to make up efficiently during unsafe conditions. They achieved better decision making in behavior level. But the limitation is that this method cannot be applied directly vehicle planning because of small amount of vehicle kinematics.

In summary, this research article comprises the effective motion planner for AV in several surrounding environment (uncertainty condition, unexpected traffic participants). This research comparative study provides good clarity for selection of best deterministic motion planner to uncertainty condition of AV. 
Journal of Electronics and Informatics (2020)

Vol.02/ No.04

Pages: 211-219

https://www.irojournals.com/iroei/

DOI: https://doi.org/10.36548/jei.2020.4.003

\section{Problem Statement}

The trajectory planning of autonomous vehicle not acquires high dimensional space still due to nonappearance of real time constraint. More over the optimal solutions are answering only pre predictable traffic and simple horse eye targets. Also these perception data are not suitable for very high speed driving and offers lag response.

\section{Proposed Solution}

The safe trajectory planning for a vehicle is that training the learning capability of vehicle with more features classification of uncertainty surrounding environment.

\section{The Oretical Approach and Discussions}

Generally the modeling for motion planning comprises planning and controlling unit in it. The planning algorithms are measuring to approximate a system behavior in reaction to feedback actions in significant functioning environments [14].

\subsection{Motion Planning}

The common methods for motion planning for self-driving will be discussing in this section. The motion planning consists of four constraints as follows;

\subsubsection{Path planning}

The road path network is representing with road corners weights equivalent to the rate of navigating the route. Initially the vehicle's decision making system must select path through the map network from source to destination. Such route can be framed as the problem of finding optimal path in the network graph. This optimal path planning stated,

Let $x$ be the configuration space of the autonomous vehicle

$$
\sum(x) \text { is set of continues function between } 0 \text { to } 1
$$

Source and destination point of travel denoted as $x_{s} \& x_{d}$ the establishing path can be noted as $E_{p}\left(x, x^{\prime}, x^{\prime \prime}, \ldots\right)$ and the degree of safe, path curve for comfort and dynamical feasible trajectory from the vehicles present to destination point [15].

Optimal Path planning

$$
\begin{aligned}
& \text { Optimal Path }=\underset{\sigma \in \sum x}{\arg \min } \text { minimum curvature } \\
& \qquad \begin{array}{c}
\sigma(0)=x_{s} \& \sigma(1) \in x_{d} \\
\sigma(\alpha) \in x_{\text {free }}
\end{array} \\
& D\left(\sigma(\alpha), \sigma^{\prime}(\alpha), \sigma^{\prime \prime}(\alpha) \ldots\right) \text { Where } \forall \alpha \in[0,1]
\end{aligned}
$$

In order to stabilize the path is that the feedback reference path and velocity will compute. The path stabilization for the model is being by pure pursuit controller. And this control is fitting on the vehicle configuration to a point on the predicted path of the vehicle $[17,15]$. The deviation distance will be computed and configured value in the path planning as optimal road map [14].

\subsubsection{Trajectory planning}

This framework concentrates in dynamic environment or constraints for autonomous vehicle. For the optimal trajectory planning consider time parameterized function $\pi(t):[0, T]$ this is configuration of vehicle in time.

$$
\begin{aligned}
& \text { Let } \prod_{\text {Optimal trajectory }}=\underset{\pi \in \Pi(x, T) \text { be set of continuous function between } 0 \text { to } T}{\arg \min } \text { dynic constraint } \\
& \qquad \begin{array}{c}
\pi(0)=x_{s} \& \pi(1) \in x_{d} \\
\pi(\alpha) \in x_{\text {free }}
\end{array}
\end{aligned}
$$

ISSN: 2582-3825 (online)

Submitted: 15.12 .2020

Accepted: 05.01.2021

Published: 13.01.2021 
Journal of Electronics and Informatics (2020)

Vol.02/ No.04

Pages: 211-219

https://www.irojournals.com/iroei/

DOI: https://doi.org/10.36548/jei.2020.4.003

$$
D\left(\pi(t), \pi^{\prime}(t), \pi^{\prime \prime}(t) \ldots\right) \text { Where } \forall t \in[0,1]
$$

Computing the stabilization for trajectory is considering an initial stability in a time varying system. This approach is considering two various types of asymptotic stability and exponential stability. The front wheel position and rear wheel position feedback is controlled by the regulated variables. The error rate is minimized by change of coordinates for steering angle as shown in figure 3.

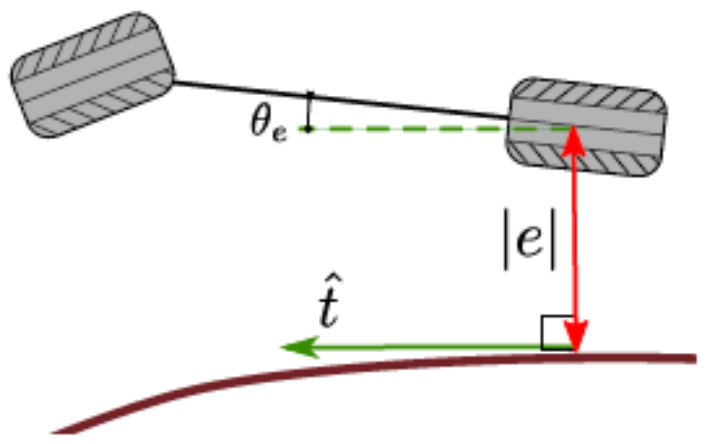

Figure 3 Wheel controller mechanisms by feedback control for steering error minimization

\section{Trajectory Tracking Control}

Lyapunov function [18] is described for this trajectory tracking control. This approach is used to refine the configuration error. This configuration error will be minimized using reference trajectory and velocity.

\subsubsection{Variational method}

The path represents as a function factorized by optimized dimensional vector. Here are lot of approaches are optimized for self-driving car with learning algorithm. This framework is aimed for optimized nonlinear continuous function. Generally the direct methods in variational approach are used to control the approximate solution to subspace of dynamic environment or constraints [3, 19].

\subsubsection{Graph search method}

The graph search method uses for set the relevant path planning for autonomous vehicle. Commonly Dijkstra algorithm uses to find the shortest paths between source to destination in the network map [20]. This method is used to build a tree for routing by given source vertex to all other vertices in the graph. The figure 4 illustrates the single track model kinematics without no slip assumption.

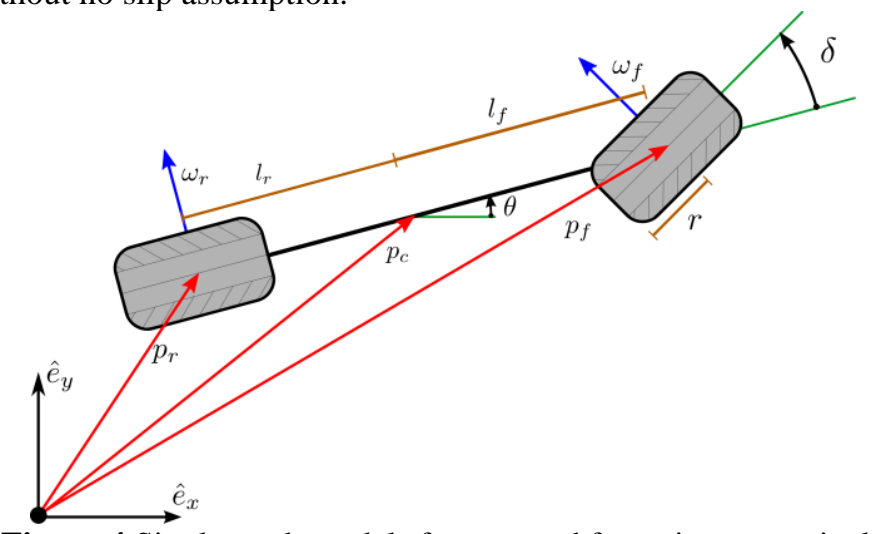

Figure 4 Single track models for rear and front tire respectively

\subsection{AI Based self-driving car}

The learning controller method is common and traditional method for AI based self-driving car.

ISSN: 2582-3825 (online) 
Journal of Electronics and Informatics (2020)

Vol.02/ No.04

Pages: 211-219

https://www.irojournals.com/iroei/

DOI: https://doi.org/10.36548/jei.2020.4.003

\subsubsection{Learning controller}

Traditional controllers will build use of a priori model composed of fastened parameters. Once robots or different autonomous systems are employed in complicated environments, such as driving, ancient controllers cannot foresee each potential situation that the system needs to deal with. In contrast to controllers with fastened parameters, learning controllers build use of training information to be stated their models over time. With every gathered batch of coaching information, the approximation of the true system model becomes a lot of correct, so facultative model flexibility, consistent uncertainty estimates and anticipation of repeatable effects and disturbances that cannot be modeled before preparation [21]. A major advantage of learning controllers is that they optimally combine ancient model based management theory with learning algorithms. This makes it doable to still use established methodologies for controller style and stability analysis, alongside a strong learning element applied at system identification and prediction level.

\subsubsection{End to End Learning control}

In the context of autonomous of autonomous driving, finish to finish learning control is outlined as an on the spot mapping from sensory information to control commands. The inputs square measure typically from a high dimensional features house (eg. pictures or fog clouds). This can be an opposition ancient process pipelines, wherever initially objects square measure detected within the input image, when that a path is planned and eventually the computed management values square measure dead. There square measure many finish to finish learning system exists finish to finish learning may also be developed as a back propagation algorithm scaled up to advanced models. Over the last number of years, the technological advances in computing hardware have expedited the usage of finish to finish learning models. The back propagation algorithmic rule for gradient estimation in deep networks is currently expeditiously implemented on parallel Graphic Process Units (GPUs). This type of process permits the coaching of enormous and complex network architectures that successively need huge amounts of coaching samples. End to End strategies are popularized within the last couple of years by NVIDIA ${ }^{\circledR}$ as a part of the PilotNet design. The approach is to coach a CNN that maps raw pixels from one front-facing camera on to steering commands [22]. The coaching information consists of pictures and steering commands collected in driving eventualities performed in a numerous set of lighting and atmospheric condition as well as on totally different road varieties. Before coaching, the information is enriched using augmentation, adding artificial shifts and rotations to the original information. The analysis is performed in two levels: Initial in simulation and second during a take a look at automobile. An autonomy performance metric represents the proportion of your time once the neural network drives the car:

$$
\text { Autonomy }=\left(1-\frac{(\text { no. of interventions }) * 6 \mathrm{sec}}{\text { elapsed time }[\mathrm{sec}]}\right) * 100
$$

An intervention is taken into account to require place once the simulated vehicle departs from the middle line by quite one meter, assumptive that half dozen seconds is that the time required by a human to retake management of the vehicle and convey it back to the required state [10].

\subsection{Motion planning based on Grid}

The grid based motion planning is generated set of continuous curvature path. In this method, uncertainty values are located in any range due to not limited working range. The initialization for motion planning is taking place here. Next the candidate evaluation is happening by check the validity of the path candidates [13]. The present cost is depends on the previous defined cost function. All the effective candidates are chosen with minimum cost of final trajectory calculation. The selection of best candidate is based on speed profile and comfort inside the autonomous vehicle.

\subsection{Proposing Approach}

The trajectory planning of autonomous vehicle not acquires high dimensional space still due to nonappearance of real time constraint. More over the optimal solutions are answering only pre predictable traffic and simple horse eye targets [10]. Also these perception data are not suitable for very high speed driving and offers lag response. The assumptions are considered during our research work on motion planning as follows,

Assumption 1: Perfect Perception

The classical localization and perception reads perfectly with stable for static obstacles and precisely prediction for dynamic obstacles.

ISSN: 2582-3825 (online)

Submitted: 15.12 .2020

Accepted: 05.01.2021

Published: 13.01 .2021 
Journal of Electronics and Informatics (2020)

Vol.02/ No.04

Pages: 211-219

https://www.irojournals.com/iroei/

DOI: https://doi.org/10.36548/jei.2020.4.003

Additionally, shape of the vehicle convolves to the map which is used for road coordinate system that indexed by station and latitude.

Assumption 2: Perfect low and high level path planning

The planned trajectory assumes low and high level path tracking executes perfectly. Additionally,

No precise trajectory knowledge of human driver; But he knows about how to drive and how to avoid the obstacle.

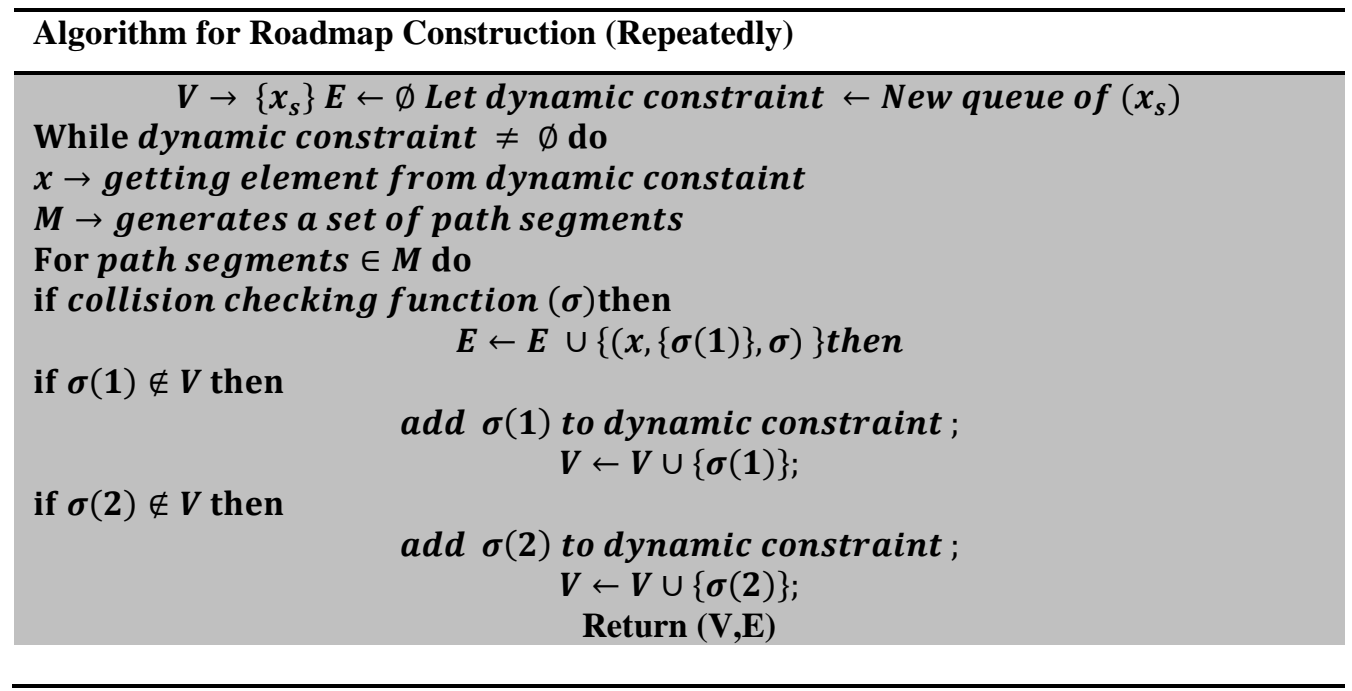

\section{Proposed Solution}

The safe trajectory planning for a vehicle is that training the learning capability of vehicle with more features classification of uncertainty surrounding environment. The motion planning algorithm will be extending the abilities of path planning and trajectory tracking control by Deep learning image classification which gives better scene understanding to autonomous vehicle. Object detection is that the problems of finding the placement of associate degree object of a certain class within the image which captured by camera. Sensible face detection and pedestrian detection are enclosed during the task. Image classification could be a problem to verify the class to which associate degree object in a picture belongs to, among predefined classes within the typical machine learning, image recognition before deep learning is not continuously optimum as a result of image options are extracted associate degree expressed exploitation an formula designed supported the data of researchers, that is termed a handcrafted feature. The figure 5 shows the path planning by self-driving car with more image recognition with scene understanding.

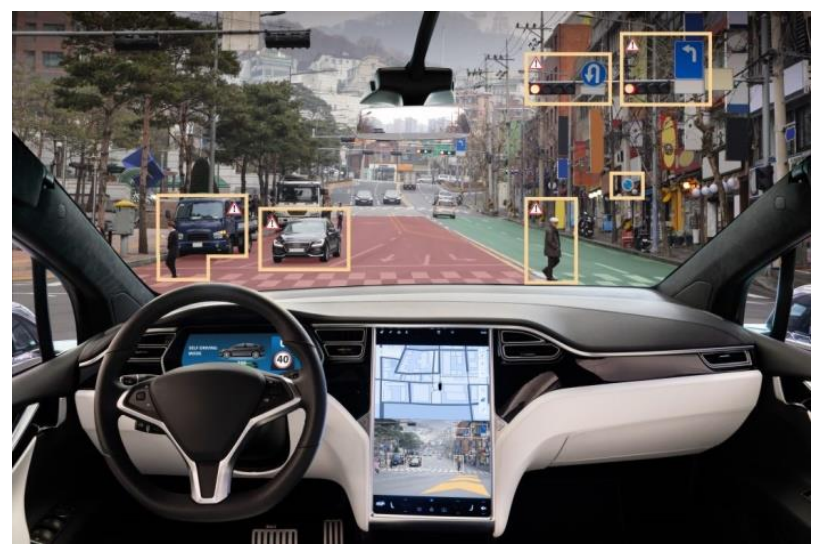

Figure 5 Path planning by Self driving car with image recognition

Convolutional neural network (CNN) which is one form of deep learning is associated degree approach for learning classification and feature extraction from coaching samples, focuses on object detection and scene 
Journal of Electronics and Informatics (2020)

Vol.02/ No.04

Pages: 211-219

https://www.irojournals.com/iroei/

DOI: https://doi.org/10.36548/jei.2020.4.003

understanding (semantic segmentation) that is shown in figure 6. Scene understanding is that the downside of understanding the scene structure in a picture [17]. Specific visual perception is that the problem of finding a selected object. By giving attributes to things with correct nouns, specific visual perception is outlined as a subtask of the final visual perception downside. CNN based end to end learning contains a downside wherever the premise of output management price is not notable. To deal with this downside, research is being conducted on associate degree approach on the judgment grounds (such as turning wheel to the left or right and stepping on brakes) that can be understood by humans. The common approach to clarify the rationale of the network higher cognitive process is a visual rationalization $[23,24,25]$.
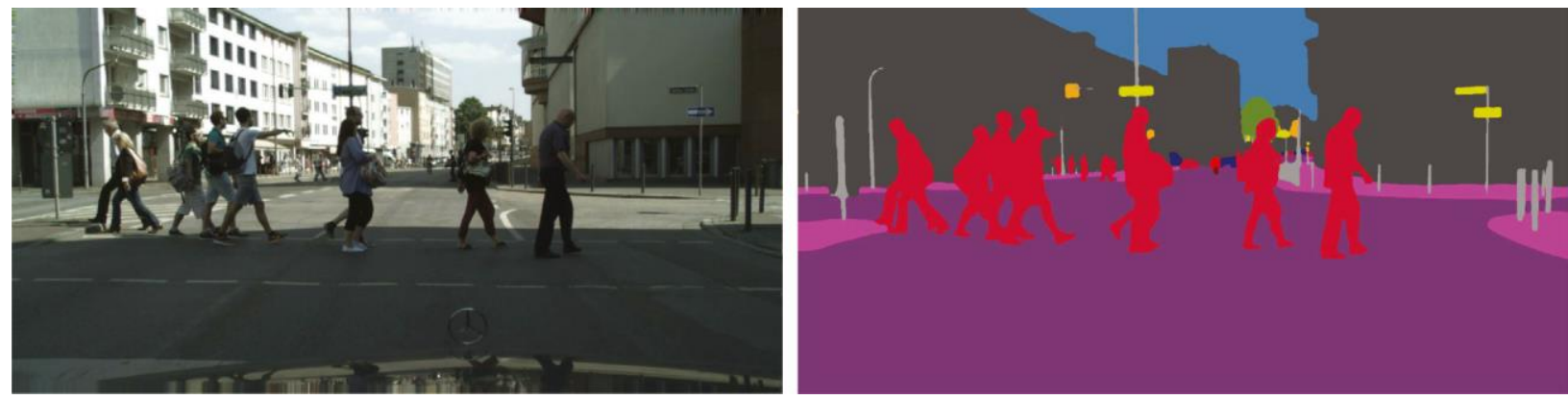

Figure 6 Example of Semantic Segmentation Results (cited from Reference [11]).

Visual rationalization technique outputs associate degree attention map that visualizes the region within which network focused as a heat map supported the obtained attention map, we can analyze and perceive the rationale of the decision making. To obtain more explicable and clearer attention map for economical visual rationalization, a number of ways are projected within the computer version field Category Activation Mapping (CAM) [24] generates attention maps by co efficient the feature maps obtained from the last convolutional layer in a very network.

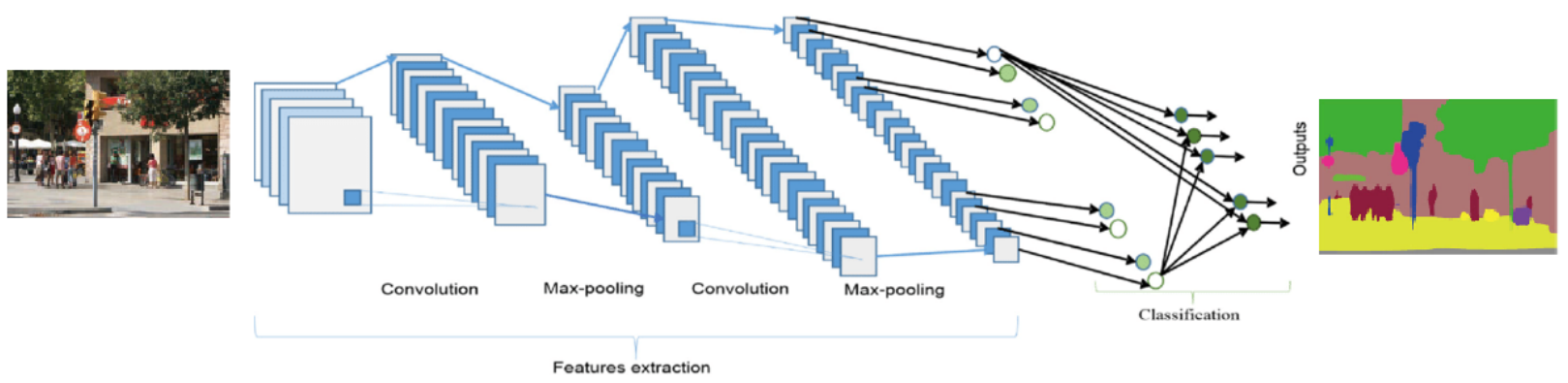

Figure 7 Image Classifications with CNN

\section{Conclusion}

This research article provides various estimate control for motion planning for autonomous vehicle. There are number of many effective feedback control algorithm are executed and stated for motion planning controller. The involvement of image recognition techniques with motion control will provide better performance. Recently, the deep learning methods are providing more effective results for control engineers and researchers. Still there is necessity of implementing an improving version motion planning algorithm to sudden uncertainty such as, the sudden changes of track, no cars in the adjacent side of the autonomous vehicles. We are suggesting the image recognition by deep learning for those instant uncertainty conditions with more effective one for AV. The visualizing the corresponding map in an AV system should be improved with artificial intelligent. The transforming from recognition results and visual details are converting into verbal details. This process is much big challenging process in the future. Many researches is conducting and not achieving sufficient accuracy and overall performance for the flexible verbal details. But these verbal details will not be necessary once the great confidence should be established between human and AV. In summary, the machine learning techniques can be applied to use better computational architectures when uncertainty condition occurs dynamically in further work. 
Journal of Electronics and Informatics (2020)

Vol.02/ No.04

Pages: 211-219

https://www.irojournals.com/iroei/

DOI: https://doi.org/10.36548/jei.2020.4.003

\section{ACKNOWLEDGEMENT}

We would like to acknowledge our Dr. Samuel Isaac, Eritrea, Mainefhi college of Engineering and Technology, for the timely guidance to this research article and he provided materials to bring this idea.

\section{REFERENCES}

[1] B. Paden, M. Čáp, S. Z. Yong, D. Yershov and E. Frazzoli, "A Survey of Motion Planning and Control Techniques for Self-Driving Urban Vehicles," in IEEE Transactions on Intelligent Vehicles, vol. 1, no. 1, pp. 33-55, March 2016, doi: 10.1109/TIV.2016.2578706.

[2] Grigorescu, Sorin \& Trasnea, Bogdan \& Cocias, Tiberiu \& Macesanu, Gigel. (2019). A survey of deep learning techniques for autonomous driving. Journal of Field Robotics. 37. 10.1002/rob.21918.

[3] W. Xu, J. Pan, J. Wei and J. M. Dolan, "Motion planning under uncertainty for on-road autonomous driving," 2014 IEEE International Conference on Robotics and Automation (ICRA), Hong Kong, 2014, pp. 2507-2512, doi: 10.1109/ICRA.2014.6907209.

[4] C. Urmson, J. Anhalt, D. Bagnell, C. Baker, R. Bittner, M. Clark, J. Dolan, D. Duggins, T. Galatali, C. Geyer, et al., "Autonomous driving in urban environments: Boss and the urban challenge," Journal of Field Robotics, vol. 25, no. 8, pp. 425-466, 2008.

[5] M. McNaughton, C. Urmson, J. Dolan, and J. Lee, "Motion planning for autonomous driving with a conformal spatiotemporal lattice," in Robotics and Automation (ICRA), IEEE International Conference on, vol. 1, pp. 48894895, 2011.

[6] H. Zhao, J. Shi, X. Qi, X. Wang, J. Jia, Pyramid scene parsing network, Proc. of IEEE Conference on Computer Vision and Pattern Recognition (2017).

[7] T. Gu and J. M. Dolan, "On-road motion planning for autonomous vehicles," in International Conference on Intelligent Robotics and Applications (ICIRA), pp. 588-597, 2012.

[8] S. Thrun, W. Burgard, D. Fox, et al., Probabilistic robotics, vol. 1. MIT press Cambridge, 2005.

[9] A. Bry and N. Roy, "Rapidly-exploring random belief trees for motion planning under uncertainty," in Robotics and Automation (ICRA), 2011 IEEE International Conference on, pp. 723-730, IEEE, 2011.

[10] J. Van Den Berg, P. Abbeel, and K. Goldberg, "Lqg-mp: Optimized path planning for robots with motion uncertainty and imperfect state information," The International Journal of Robotics Research, vol. 30, no. 7, pp. 895913, 2011.

[11] M. Althoff, O. Stursberg, and M. Buss, "Safety assessment of autonomous cars using verification techniques," in American Control Conference, 2007. ACC’07, pp. 4154-4159, IEEE, 2007.

[12] M. Althoff, O. Stursberg, and M. Buss, "Model-based probabilistic collision detection in autonomous driving," Intelligent Transportation Systems, IEEE Transactions on, vol. 10, no. 2, pp. 299-310, 2009.

[13] J. Wei, J. M. Dolan, J. M. Snider, and B. Litkouhi, "A point-based mdp for robust single-lane autonomous driving behavior under uncertainties," in Robotics and Automation (ICRA), 2011 IEEE International Conference on, pp. 2586-2592, IEEE, 2011.

[14] A. Artuñedo, J. Villagra, J. Godoy and M. D. d. Castillo, "Motion Planning Approach Considering Localization Uncertainty," in IEEE Transactions on Vehicular Technology, vol. 69, no. 6, pp. 5983-5994, June 2020, doi: 10.1109/TVT.2020.2985546.

[15] Gu, Tianyu \& Dolan, John. (2012). On-Road Motion Planning for Autonomous Vehicles. 7508. 588-597. 10.1007/978-3-642-33503-7_57.

[16] H. Yin and C. Berger, "When to use what data set for your self-driving car algorithm: An overview of publicly available driving datasets," 2017 IEEE 20th International Conference on Intelligent Transportation Systems (ITSC), Yokohama, 2017, pp. 1-8, doi: 10.1109/ITSC.2017.8317828.

[17] Fujiyoshi, Hironobu \& Hirakawa, Tsubasa \& Yamashita, Takayoshi. (2019). Deep learning-based image recognition for autonomous driving. IATSS Research. 43. 10.1016/j.iatssr.2019.11.008.

[18] A. Chakarov, A. Nori, S. Rajamani, S. Sen, and D. Vijaykeerthy, "Debugging Machine Learning Tasks," arXiv preprint arXiv:1603.07292, 2018.

[19] Frazzoli, Emilio \& Emilio,. (2005). Robust hybrid control for autonomous vehicle motion planning. Proceedings of the IEEE Conference on Decision and Control.

[20] Y. Wang, W.-L. Chao, D. Garg, B. Hariharan, M. Campbell, and K. Weinberger, "Pseudo-LiDAR from Visual Depth Estimation: Bridging the Gap in 3D Object Detection for Autonomous Driving," in IEEE Conf. on Computer Vision and Pattern Recognition (CVPR) 2019, June 2019.

ISSN: 2582-3825 (online)

Submitted: 15.12 .2020

Accepted: 05.01.2021

Published: 13.01.2021 
Journal of Electronics and Informatics (2020)

Vol.02/ No.04

Pages: 211-219

https://www.irojournals.com/iroei/

DOI: https://doi.org/10.36548/jei.2020.4.003

[21] C. J. Ostafew, J. Collier, A. P. Schoellig, and T. D.Barfoot, "Learning-based Nonlinear Model Predictive Control to Improve Vision-based Mobile Robot Path Tracking," Journal of Field Robotics, vol. 33, no. 1, pp. 133-152, 2015. [22] Li, Jun \& Cheng, Hong \& Guo, Hongliang \& Qiu, Shaobo. (2018). Survey on Artificial Intelligence for Vehicles. Automotive Innovation. 1. 10.1007/s42154-018-0009-9.

[23] K. Mori, H. Fukui, T. Murase, T. Hirakawa, T. Yamashita, H. Fujiyoshi, Visual explanation by attention branch network for end-to-end learning-based self-driving, Proc. of IEEE Intelligent Vehicles Symposium (9-12 June 2019) https://doi.org/10.1109/ IVS.2019.8813900.

[24] B. Zhou, A. Khosla, A. Lapedriza, A. Oliva, A. Torralba, Learning deep features for discriminative localization, Computer Vision and Pattern Recognition 2016, pp. 2921-2929.

[25] R.R. Selvaraju, M. Cogswell, A. Das, R. Vedantam, D. Parikh, D. Batra, Grad-CAM: visual explanations from deep networks via gradient-based localization, International Conference on Computer Vision 2017 , pp. 618-626.

ISSN: 2582-3825 (online)

Submitted: 15.12.2020

Accepted: 05.01.2021

Published: 13.01.2021 